\title{
Incremental Update of a Digital Twin of a Production System by Using Scan and Object Recognition
}

\author{
Markus SOMMER ${ }^{\mathrm{a}}$, Josip STJEPANDIĆ ${ }^{\mathrm{b} 1}$ and Sebastian STOBRAWA \\ ${ }^{a}$ isb - innovative software business GmbH, Germany \\ ${ }^{b}$ PROSTEP AG, Germany \\ ${ }^{c}$ Leibniz University Hannover, Institute of Production Engineering and Machine Tools, \\ Germany
}

\begin{abstract}
While production plants are subjects of frequent change, for instance due to changed processes, new products or new machine tools, the process plans must be subsequently updated. This generally affects all planning processes for production management and thus in particular also modern planning methods such as the Digital Twin of a production system. The simulation of production processes using a Digital Twin is a promising means for prospective planning, analysis of existing systems or process-parallel monitoring. However, many companies, especially small and medium-sized enterprises, do not apply the technology, because the generation of a Digital Twin is cost-, time- and resource-intensive and IT expertise is required. Supposed that the process of generation of the Digital Twin was completed once using scans and deep learning applied to a point cloud of a production system, this paper describes a conceptual approach to provide an incremental update of this Digital Twin, as often as necessary. The solution alternatives are presented and discussed, in particular the use of flexible devices $\left(360^{\circ}\right.$-camera) for the object acquisition. A particular attention is given to the integration of the entire change process for updating an existing Digital Twin.
\end{abstract}

Keywords. Digital Twin, Digital Factory, Object Recognition, Indoor Object Acquisition, Simulation, Transdisciplinary Engineering

\section{Introduction}

The Digital Twin is the surrogate of a physical object or system, which can be a product, a production system, but also a company or a process. As a frequently used buzzword which connects research and commercial applications, Digital Twin can be found also in further, non-technical domains such as medicine, economy or society [1]. The Digital Twin connects virtual planning and development models with the real world of products and production systems in order to obtain insight of the system and its state as well as behavior. A vision in the sense of Industry 4.0 is that technically complex systems can control themselves autonomously and behave more intelligently using digital algorithms, virtual models and status information [2]. Therefore, the virtual representation of the Digital Twin enables in the realisation of this vision by providing the real system with a digital operational layer on which the autonomous and intelligent algorithms can execute.

\footnotetext{
${ }^{1}$ Corresponding Author, Mail: josip.stjepandic@prostep.com.
} 
The mutual functional relationships of products or production systems are determined based on customer requirements and taking into account a large number of legal requirements in product planning and development. Without knowledge of these relationships, the operating data that the real asset records in its later product life cannot be interpreted correctly. If it is not known how a machine or system should actually work, it is not possible to unequivocally identify the causes of deviations from this desired state or behavior and enforce appropriate countermeasures. Knowing the history of its origins is also important in order to be able to assess the reason, for example, of a bearing failure and which other machines could also be affected by the problem [2]. With such information, the behavior of a technical system can be easily predicted.

One of the distinctive dimensions of Digital Twin is the time of creation. The approach to create the virtual model first or synchronouosly with the physical counterpart is sufficiently supported by modern software tools. The opposite way which consists of digitalization or reverse engineering of an existing environment is not that well covered by methods and tools, and therefore subject of various research activities. In previous research of these authors, an approach for generation of Digital Twin of manufacturing in a built environment was developed which addresses the entire factory hall [3]. With fast scans of the shop floor and subsequent object recognition, the production layout (e. g. size and location of the objects) and the production semantic (e. g. machine types, transport routes) can be recorded as automated as possible and visualized true to scale in digital models [4]. This allows a Digital Twin of an already existing production system to be set up quickly and thus cost-effectively. It should be emphasised at this point that the Digital Twin is built up initially and the structure is thus fixed [5].

Production systems are constantly being rescheduled and rebuilt to increase their productivity or to use them to produce other products [6]. There are therefore many good reasons for operators to precisely record the current status of their plant and production system. This actual status never exactly corresponds to the planning status, because there are often deviations during the construction of the system or many details are not planned at all. That is why many operators try to bundle the information on their existing systems in a Digital Twin [7]. However, the operators are faced with the question of how they can set up this digital surrogate with a reasonable expenditure of time and money [8].

This need is in particular supported by the process simulation as a core element of the digital factory and is becoming increasingly important for small-and-medium enterprises as a result of developments in the area of digitisation [3]. Nevertheless, current studies prove that the use of simulation models for production systems in small and medium-sized enterprises is still not standard [4]. This is particularly due to the fact that the costs for setting up and operating the Digital Twin are not transparent and tend to be estimated as too high. IT knowledge is also lacking, so that the benefits cannot be correctly estimated or there is a reluctance to implement the Digital Twin [5]. At this point, it is also often critical that a one-off investment in a Digital Twin is seen as unsafe, as companies are aware that a continuous updating process is necessary to keep the Digital Twin up to date and valid [6]. Small companies have small tasks, e.g. replacement of a machine by a new one, which do not require the acquisition of the entire hall. Thus, a flexible solution for a intermediate, iterative update of the Digital Twin is necessary.

The remainder of this paper is structured as follows: In Section 1, the problem statement is expressed. Section 2 illustrates the importance and options in the data acquisition. Section 3 presents the solution concept for iterative update which is further detailled and structured in Section 4. Finally, Section 5 summarizes the conclusions and outlook. 


\section{Problem statement}

In general, different processes are conceivable for setting up a Digital Twin. Often, a Digital Twin is built by people with the expertise of the corresponding software tools manually. For this purpose, these people must be provided with the relevant information on the production system to be mapped so that they can create the Digital Twin. However, the authors of this article have developed and researched a procedure for efficient generation of a Digital Twin over the last two years, so that this will be presented here as a feasible alternative [8]. The further explanations are also oriented towards this procedure and based on it. The efficient, generic approach for generation of the Digital Twin of an existing production system is depicted in Figure 1. During the initial setup, the operator of a plant provides his facility (1) which is being scanned (2) by a commercial device [3]. The resulting point cloud is transferred to CAD modeling which creates a CAD assembly of the production system or the entire plant based on the point cloud. The most challenging task in this process is the object recognition in the cloud based on a CAD library [9]. The equipment characteristics are collected by a different way to provide the initial setup. This setup means that a Digital Twin can be created once with the method with no possibility for further adjustment of the Digital Twin is carried out after the method has been completed.

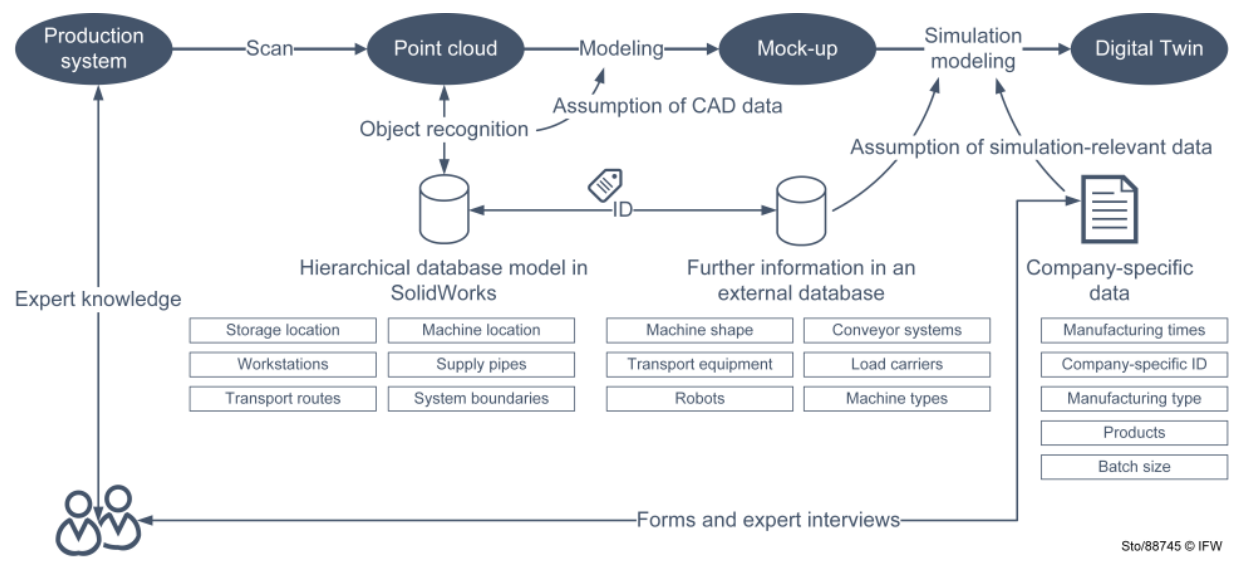

Fig. 1. Approach for generation of the Digital Twin of production system

The exploitation of the Digital Twin can take place from this point on, but production systems often change during its lifecycle [10]. The (digital) twinning implies an immediate, instant change of the digital surrogate in case of change of the corresponding physical part and vice versa. The following constraints are especially important, although further modifications to the production system are possible:

- Adjustment activities change the production sequences, which in turn changes processing times, for example. This is caused by the inherent mission of production management to continuously increase productivity through minor adjustments.

- The production schedule may change over time. This input variable for the production system, meaning which product is to be produced at which time, is usually subject to constant alterations. 
- New products or product variants impose various adjustments to be made to the production processes or new ones to be developed.

- Besides products, new machines or new transport equipment may be added to the production system or old machines may be removed from the production system.

- The layout of the production system can change by repositioning objects in the production hall.

- Often are such modifications cummulated.

The aforementioned changes mean that the resulting Digital Twin must be updated whenever necessary [10]. However, updating the Digital Twin is as important as a change relevant for the simulation results can occur. Three general cases are explained here as illustration of this change and update, which pose different levels of complexity and effort for updating and should therefore be considered as separate cases. In ascending order of complexity, these cases are shown in the Table 1.

Table 1. Cases of update of the Digital Twin.

\begin{tabular}{ll}
\hline Case & Description/Content \\
\hline Parameter-based & $\begin{array}{l}\text { The changes in the real system lead to the requirement that the Digital Twin } \\
\text { can remain in its current form, but that single parameters have to be updated. } \\
\text { It can be provided remotely. No additional object acquisition is necessary. }\end{array}$ \\
\hline Structural partial & $\begin{array}{l}\text { The changes in the real system require an adjustment of the Digital Twin, but } \\
\text { the general structure can be preserved so that a new scan is not necessary or } \\
\text { only isolated areas have to be re-recorded. }\end{array}$ \\
\hline Structural full & $\begin{array}{l}\text { The changes in the real production system are so extensive that a new } \\
\text { recording is required and the Digital Twin has to be completely recreated. }\end{array}$ \\
\hline
\end{tabular}

A parameter-based and (entire) structural updates suppose re-use or repeat of the generation of the Digital Twin with the already developed method [4]. For the partial update, the same metod can be re-used too, but with regard to a significantly reduced scope. It implies an appropriate object acquisition method for a reduced space which considers the specific local circumstances (accessability, impact of darkness, dirt, dust, occlusion).

\section{Object acquisition}

The flexibility and speed how the production system is being acquired has the particular importance on the affordability of this approach. The most used acquisition devices are the scanners which can be used as either fixed or mobile devices. However, new approaches becomes known just recently which use end-user commercial camera for shape acquisition [11].

The comparison between the terrestrical laser scanner and the $360^{\circ}$-camera is shown in Table 2. As a mature technology, the scanner discovers advantages in accuracy and availability of rough data. The comparison of the whole range of criteria shows that the alternative with the $360^{\circ}$-camera is the superior option [12]. 
Table 2. Comparison between terestrical laser scanner and $360^{\circ}$-camera.

\begin{tabular}{lll}
\hline \multicolumn{1}{c}{ Characteristic } & Terestrical Laser Scanner & $\mathbf{3 6 0}^{\circ}$-Camera \\
\hline Scan duration (e. q. $300 \mathrm{qm})$ & One hour (six scans) & Three minutes \\
\hline Invest costs & $80,000 €$ & $500 €$ \\
\hline Qualification of the scan operator & Expert & Everybody \\
\hline Traveling expenses & Yes & No \\
\hline Availability of the point cloud & 0.5 hour & One day \\
\hline Accuracy & $\begin{array}{l}\text { One millimeter per ten } \\
\text { meters }\end{array}$ & $\begin{array}{l}\text { Ten millimeters per ten } \\
\text { meters }\end{array}$ \\
\hline
\end{tabular}

In comparison with a scanner, a commercial $360^{\circ}$-camera acquires the video of a scene. This video must be translated into the point cloud in a subsequent process which can be performed offline. The 3D-reconstruction pipeline is shown in Figure 2. After the $360^{\circ}$-video is uploaded in the cloud, a process starts which extracts the singular images from the video and translates these in a point cloud. The particular challenge lies in the registration (the mutual localisation of singular images in the space) and the handling of light reflections on the bright surfaces.

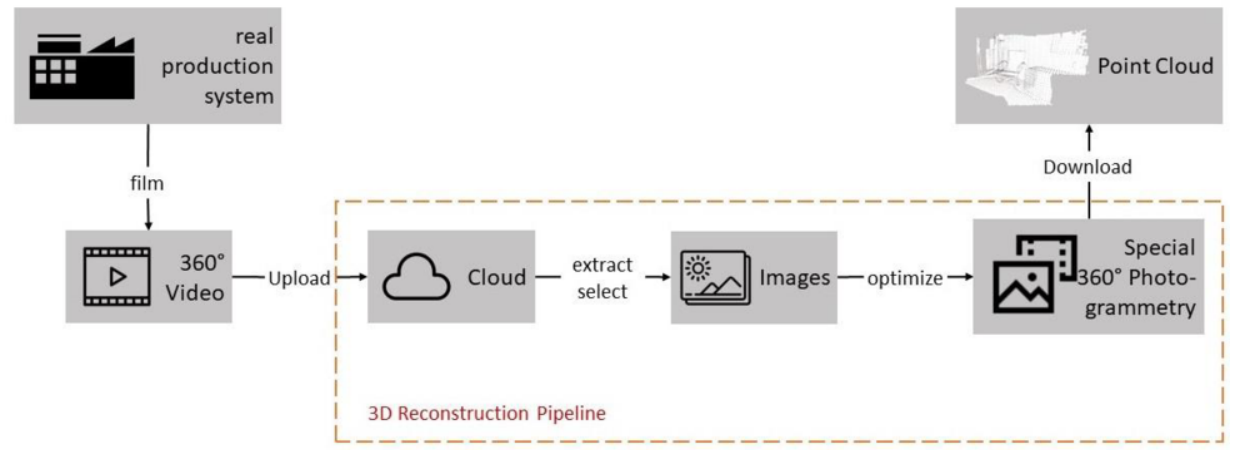

Fig. 2. 3D reconstruction pipeline based on $360^{\circ}$ video

This methods is conventient especially for smaller rooms and tasks which occuring during a update.

\section{Parameter-based update}

The implementation of the updates described in Table 1 primarily relies on the extent of the required change. The first case mentioned in Table 1 is for example when, as mentioned above, the production schedule is changed or adjustments to the production system lead to altered processing times. Moreover, new products that do not lead to structural modifications of the production system can also be addressed with this case. It is therefore a matter of adjusting parameters of the Digital Twin, such as processing or set-up times. 
A useful addition for this case is the connection of the Digital Twin to the existing IT systems, especially Enterprise Resource Planning (ERP) or Manufacturing Execution Systems (MES). However, a connection to machine or operating data is also reasonable at this point, as for example explained by Donhauser et al. [13]. With this interface connection to ERP or MES, information about products, production schedules, work plans, machining and set-up times, or similar can be transmitted directly to the Digital Twin [14]. The updating of the Digital Twin is carried out automatically here, so that this process does not require any further handling and thus provides a natural practical extension to the basis method a kind of living Digital Twin.

The simplest update process described here, which only involves parameter adjustment, is thus achievable in the next development steps. The basic functionalities are considered in the current method. However, it should be noted at this point that due to the large number of different MES and ERP, a generic solution can only be achieved with standardised transfer protocols.

\section{Structural partial update of the Digital Twin}

A much more far-reaching updating process occurs when, in addition to the parameters of the Digital Twin, the structure must also be adapted. This is always the case when objects, such as machines or material handling, are added, removed or displaced in the real production system. That is a typical case in the real life business [10]. However, this case can also arise in the case of comprehensive changes to products or the production schedule, if this results in major modifications to the production system that cannot be mapped with a pure parameter adjustment of the Digital Twin (Figure 3).

A structural update can of course be carried out by people who have expertise in the simulation software. However, this manual process would be costly as well as fault-prone and the corresponding IT expertise must be available. A gap between the model of factory and the simulation model would arise. In terms of the basis method used here, an efficient method would therefore lead to a better result, since again no IT expertise is needed, the effort is low, and the costs for the update are predictable.

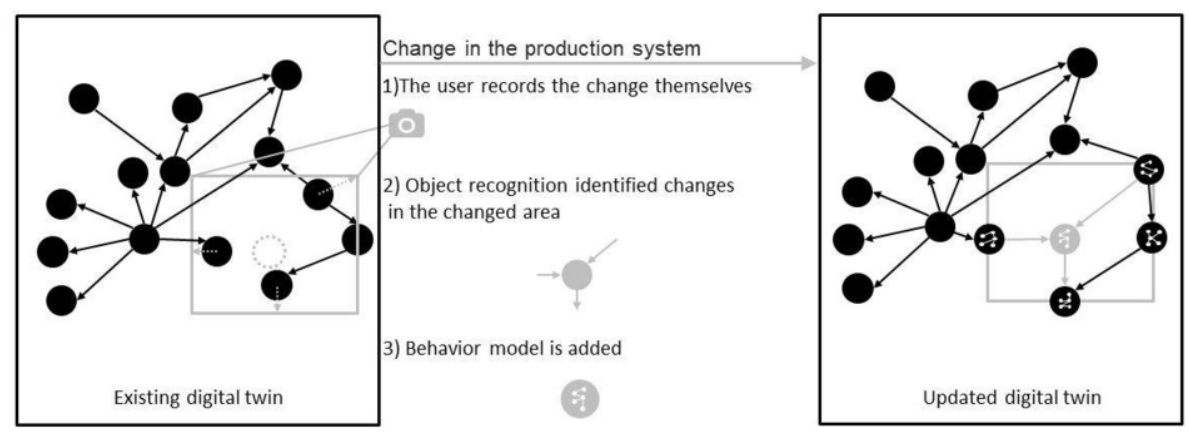

Fig. 3. Approach for the structural update of the Digital Twin

In order to conduct a real update of the Digital Twin, an extension of the method is necessary for the next development steps. This method has its particular workflow and 
consider a significant change in the object acquisition. Figure 3 provides an overview of the research approach.

The approach consists of three key characteristics, shown in the Figure 3, which are needed to proceed from a current Digital Twin (left hand side of the figure) to an updated Digital Twin (right hand side of the figure).

At first, the change in the real system should be preferably recorded by the customer himself which is particularly beneficial if the customer is located far abroad. This means that if, for example, a machine is moved to a different location in production hall or a new machine is added to the production system, only this area of particular interst is acquired. In this way, the overall effort is being reduced to a few objects of interests. To be able to do this by a customer, a simple process with a simple easy-to-use device is needed. Here, an application for consumer camera or common smartphones is developed with which a production area can be recorded as a $360^{\circ}$-video (Figure 2). Photogrammetry is used to generate the required point cloud. During the recording, the customer receives augmented reality feedback on whether the respective area has been correctly recorded by displaying objects in green on the mobile phone display if they have been captured. Finally, the recording can be sent directly to the offices that will further process the recording. For this purpose, the secure data transfer must be used [20]. The volume of data which is being generated and transferred here is significantly lower than for scan of the entire production system. If the initial result is not sufficient, this procedure can be repeated as often as necessary. Accordingly, a simple application for a consumer camera or a smartphone is created here that enables the client to take the scan of the changed areas himself. The service offered here is a lean process that can be carried out quickly without the need to work on the customer's premise.

At second, the basis method for the object recognition based on Convolutional Neural Networks should be applied in the changed areas and the adaptation of the Digital Twin. It belongs the entire process which must be adapted so that single areas from the Digital Twin can be segmented and modified. This poses a particular challenge because the edges between the existing and the modified areas have to be designed to fit each other. This issue must be resolved by an additional registration. In addition, however, this new updating method is quite similar to the method already explained for the initial creation of the Digital Twin. Here, too, a point cloud is first created from the scan (but here with a changed role by the customer). Subsequently, object recognition identifies new objects and inserts them into the CAD model, which in a further step is transferred to the simulation software to adjust the Digital Twin.

At third, if the method is able to deal with small sections of the production systems (e. g. discrete Digital Twin), it can be extended in that matter that the Digital Twin is being enriched by behavioural models as an advanced stage of the parameter-based updating of the Digital Twin. The core idea is that behavioural models are stored for the single objects, especially machine tools. These behavioural models represent the functions and capabilities of the objects, so that changed conditions, such as new products, wear, malfunctions, etc., are directly represented by the models and do not require any further updating process for the Digital Twin. Accordingly, this aspect prevents few further requirement for updating processes for the Digital Twin.

With the three characteristics presented, there is therefore an efficient incremental updating process that represents a sensible extension of the existing method. A scalable Digital Twin of manufacturing is created in short time with low effort, in which behavioural models of machines are embedded. Easy update facilitates the simulation of much more variants, making the production planning and control of industrial companies 
more reliable, efficient and comprehensive [15]. The resulting Digital Twin becomes more accurate through parameterisation from the machine level and requires fewer updating processes.

\section{Structural update of the Digital Twin}

For the last case of an update process, it is assumed that the changes in the production system are so comprehensive that partial updates of single areas cannot be carried out with a reasonable effort. Such cases occur in the context of fundamental organisational changes to the production system or when a far-reaching restructuring of the system is carried out [16].

The partial update process described in the previous section would then be too costly, as the additional effort required to assemble existing and updated parts of the Digital Twin exceeds the savings. Accordingly, in such a case, a renewed application of the basis method for the entire hall or plant as a new case would be recommended. This means that the complete process would be repeated: Scan of the factory floor and recreation of the Digital Twin with the upstream object recognition.

It should be emphasised at this point, however, that most steps can be shortened here, since an initial creation process already exists. Accordingly, company-specific data on products, for example, no longer needs to be fundamentally re-entered, but can be taken over from previous processes. Another useful extension is the integration of the machine models. This additional aspect then prevents the requirement for further updates [17]. The accuracy of object recognition would be higher based on more trainings performed.

\section{Discussion}

As presented in this paper, the Digital Twin of production system could be improved further in term of flexibility, availability and timeliness [18]. Based on technological improvements in basis disciplines (camera hardware, computer vision, deep learning, localization algorithms), the vision of a real-time recognition, localization, and simulation of complex processes in a factory is moving even closer, although a mobile all-in-one application is not achievable at this time.

A tremendous move to the mobile acquisition devices, in particular to the spherical camera, becomes obvious. Its handling is easy for untrained personal too and threedimensional reconstruction from videos and images is robust. The commercial spherical cameras achieve even higher resolutions and already meet the demand for sufficient resolution of homogeneous structures. Apart of localization, which is an inherent issue for all mobile devices, cameras struggle with a higher level of noise which become visible quickly on white walls or other homogenous surfaces [12]. In the reconstruction, these are not a straight but sinusoidal waves become visible [19].

With a spherical camera and a suitable image processing pipeline, a 3D-model can be created fast and efficiently by simple means, if the the highest accuracy of the model is not required. A further step would be the reconstruction of gaps within the 3D model at a later stage. This would simplify the merge with the basis model [20].

Object recognition is subject of intensive research in field of computer vision with remarkable advancements. Objects with distinctive features can be recognized timely, 
easily and reliably. The current challenge is more to select and adapt an appropriate, fast and reliable approach for object recognition rather than to develop a new one. The affordability of the recognition could be dramatically improved by further publicly available object databases [21].

The moment can be hardly predicted when 3D recognition methods become available on real-time applications, including mobile devices. Majority of deep learning libraries are designed for desktop operating systems, but some libraries also provide support for mobile operating systems. It heavily depends on further development on deep learning, in particular to accelerate the training procedure which is a limitation factor at this time [21].

The proposed method for update is the basis for an incremental, semi-automatic model setup for simulations of production systems. With this approach, it is possible to maintain a generic method with which any production system can be transferred into a simulation model [22]. This procedure allows the required information to be stored in the simulation software and to be accessed in the model construction with an almost instant actuality.

\section{Conclusions and outlook}

This paper describes a conceptual approach for the incremental update of the Digital Twin of an existing production system which is a step ahead for the frequent implementation of the Digital Twin. Digital Twins are a powerful means to optimize the processes in the manufacturing industry using its high-fidelity digital surrogate. This paper contributes a concept how to resolve the frequent issue of intermediate disparity between the virtual model and the physical part. With support of deep learning, the twinning becomes almost continuous.

Digital Twin is an encompassing approach of digitization for tackling complex, realworld problems. Digital Twin poses a huge potential which up to now was primarily recognized in the manufacturing industry but also in healthcare, society, and economics [1]. The Digital Twin is also an important approach in overarching earlier approaches. In essence, multiple disciplines, multiple functional roles, and multiple stakeholders need to collaborate in the processes making up the engineering, healthcare, societal, or economic systems.

The synchronous availability of the digital and the physical product alone brings interoperability into play as an indispensable prerequisite. It is enforced by dynamic interaction between technical and social characteristics of new product, process, and service development. Considering the human factor, e. g. human-machine interaction, a Digital Twin becomes part of a social-technical system. This moves the scope of Digital Twin in the area of transdisciplinary engineering [23].

While data are the fuel of Digital Twin, the main challenge lies in a seamless integration of methods and tools that are suitable to support the dynamic and evolving nature of the modern production system that need to be developed including the development system itself. The lack of standardization is particularly significant because it is unlikely that a complex system will come from a single source in a challenging environment. As a tool which can have many sub-components spread across collaborators and industry partners, developing regulations and security mechanisms is imperative for widespread of adoption of Digital Twin to overcome the concerns regarding data sharing. 


\section{References}

[1] C.K. Lo, C.H. Chen, R.Y. Zhong, A review of digital twin in product design and development, Advanced Engineering Informatics, Vol. 48, 2021, 101297.

[2] L. Wagner, Kein Digital Twin ohne digitale Durchgängigkeit. Zeitschrift für den wirtschaftlichen Fabrikbetrieb, 2020, Band 115, pp. 43-45, DOI: 10.3139/104.112315.

[3] M. Sommer, J. Stjepandić, S. Stobrawa and M. von Soden, Automatic Generation of Digital Twin Based on Scanning and Object Recognition, Adv. in Transdisciplinary Engineering, Vol. 10, 2019, pp. 645-654.

[4] M. Sommer, J. Stjepandić, S. Stobrawa and M. von Soden, Automated generation of a digital twin of a manufacturing system by using scan and convolutional neural networks, Advances in Transdisciplinary Engineering, 2020, 12, pp. 363-372.

[5] B. Denkena, M.-A. Dittrich, S. Stobrawa, J. Stjepandić, Efficient generation of a digital twin using object detection for data acquisition and XML-interface for model creation, Procedia CIRP, 2020, 93, 274-279.

[6] R. Wallis, J. Stjepandic, S. Rulhoff, F. Stromberger, J. Deuse, Intelligent utilization of digital manufacturing data in modern product emergence processes, Advances in Transdisciplinary Engineering, Vol. 1, 2014, pp. 261-270.

[7] S. Wenzel, T. Peter, Prozess-Simulation in kleinen und mittleren Unternehmen mittels des Bausteinkastensystems KMUSimMetall. In: Wenzel, S.; Peter, T. (Eds.): Simulation in Produktion und Logistik. Kassel: university press 2017, pp. 433-442.

[8] M. Sommer, J. Stjepandić, S. Stobrawa and M. von Soden, Improvement of factory planning by automated generation of a digital twin, Advances in Transdisciplinary Engineering, Vol. 12, 2020, pp. 453-462.

[9] E. Ostrosi, J. Stjepandić, S. Fukuda and M. Kurth, Modularity: New trends for product platform strategy support in concurrent engineering, Advances in Transdisciplinary Engineering, 2014, Vol. 1, pp. 414423.

[10] J. Stjepandić, M. Sommer, S. Stobrawa, Digital Twin: Conclusion and Future Perspectives, in J. Stjepandić et al. (eds.) DigiTwin: An Approach for Production Process Optimization in a Built Environment, 2021, Springer Nature, in press.

[11] D. Moon, S. Chung, S. Kwon, J. Seo, J. Shin, Comparison and utilization of point cloud generated from photogrammetry and laser scanning: 3D world model for smart heavy equipment planning, Automation in Construction, Vol. 98, 2019, pp. 322-331.

[12] M. Sommer, K. Seiffert, Scan Methods and Tools for Reconstruction of Built Environments as Basis for Digital Twins, in J. Stjepandić et al. (eds.) DigiTwin: An Approach for Production Process Optimization in a Built Environment, 2021, Springer Nature, in press.

[13] T. Donhauser, U. Endter, T. Schmidt, L. Baier, P. Schuderer, J. Franke, Echtzeitabbild der Produktion. wt werkstattstechnik, 110 (2020) 4, pp. 214-219.

[14] O. Kuhn, H. Liese and J. Stjepandic, Methodology for knowledge-based engineering template update, IFIP Advances in Information and Communication Technology, Vol. 355, Springer, Berlin Heidelberg, 2011, pp. 178-191.

[15] B. Denkena, K. Doreth, M. Köller, S. Wilmsmeier, F. Winter, F.: Simulationsbasierte kombinierte Instandhaltungs- und Produktionsplanung, G. Mayer, C. Pöge, S. Spieckermann, S. Wenzel, (eds.) Ablaufsimulation in der Automobilindustrie, Springer Vieweg, 2020, pp. 261-273.

[16] B. Denkena, S. Stobrawa, M. Sommer, J. Stjepandic, M. Von Soden, Production planning using the digital twin | [Effiziente Erstellung eines digitalen Zwillings zur Verwendung im Produktionsmanagement; Produktionsplanung mit dem digitalen Zwilling], WT Werkstattstechnik, 2020, 110(10), pp. 661-665.

[17] B. Denkena, S. Wilmsmeier, S. Hauck, Adaptive Simulationsmodelle - Integration von Maschinendaten in Materialflusssimulationen. ProductivITy, 22 (2017) 3, pp. 37-39.

[18] B. Denkena, M.-A. Dittrich, S. Stobrawa and J. Stjepandić, Automated Generation of a Digital Twin Using Scan and Object Detection for Data Acquisition, in: M. Putz and A. Schlegel (Hrsg.) Simulation in Produktion und Logistik 2019, Wissenschaftliche Scripten, Auerbach, 2019, pp. 49-60.

[19] Z. Ma, S. Liu, A review of 3D reconstruction techniques in civil engineering and their applications, Advanced Engineering Informatics, Vol. 37, 2018, pp. 163-174.

[20] F. Xue, W. Lu, K. Chen, C.J. Webster, BIM reconstruction from 3D point clouds: A semantic registration approach based on multimodal optimization and architectural design knowledge, Advanced Engineering Informatics, Vol. 42, October 2019, Article number 100965.

[21] B. Salem, J. Stjepandić and S. Stobrawa, Assessment of Methods for Industrial Indoor Object Recognition, Advances in Transdisciplinary Engineering, Vol. 10, 2019, pp. 390-399.

[22] G. da Silva Serapião Leal, W. Guédria, and H. Panetto, An ontology for interoperability assessment: A systemic approach, Journal of Industrial Information Integration, Vol. 16, 2019, 100100.

[23] N. Wognum, C. Bil, F. Elgh, M. Peruzzini, J. Stjepandić and W.J.C. Verhagen, Transdisciplinary engineering research challenges, Advances in Transdisciplinary Engineering, 2018, Vol. 7, pp. 753-762. 